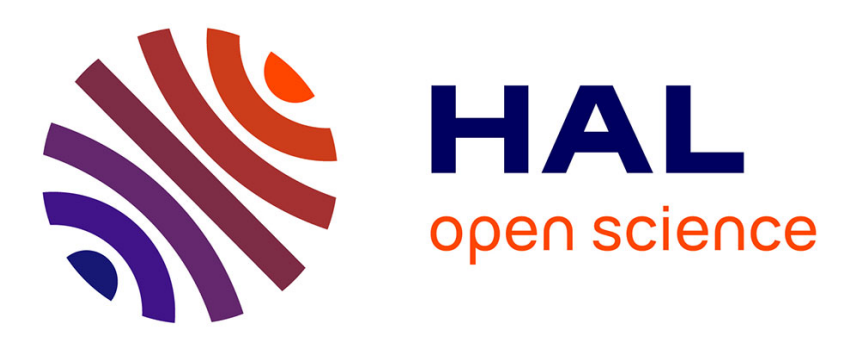

\title{
Nutrient control of hunger by extrinsic gastrointestinal neurons.
}

Gilles Mithieux

\section{To cite this version:}

Gilles Mithieux. Nutrient control of hunger by extrinsic gastrointestinal neurons.. Trends Endocrinol Metab, 2013, epub ahead of print. 10.1016/j.tem.2013.04.005 . inserm-00835940

\section{HAL Id: inserm-00835940 https://www.hal.inserm.fr/inserm-00835940}

Submitted on 20 Jun 2013

HAL is a multi-disciplinary open access archive for the deposit and dissemination of scientific research documents, whether they are published or not. The documents may come from teaching and research institutions in France or abroad, or from public or private research centers.
L'archive ouverte pluridisciplinaire HAL, est destinée au dépôt et à la diffusion de documents scientifiques de niveau recherche, publiés ou non, émanant des établissements d'enseignement et de recherche français ou étrangers, des laboratoires publics ou privés. 
1 NUTRIENT CONTROL OF HUNGER BY EXTRINSIC GASTROINTESTINAL 2 NEURONS

3

4 Gilles Mithieux ${ }^{1.2 .3}$

5

$6{ }^{1}$ Inserm U855, Faculté de Médecine Lyon-Est « Laennec », 69372 Lyon Cedex 08, France

$7 \quad{ }^{2}$ Université Lyon 1, 69622 Villeurbanne, France

$8 \quad{ }^{3}$ Université de Lyon, 69008 Lyon, France

9

10 Corresponding author: Mithieux Gilles: gilles.mithieux@inserm.fr 11 12 


\section{ABSTRACT}

The neural sensing of nutrients during food digestion plays a key role in the regulation of hunger. Recent data have emphasized that the extrinsic gastrointestinal nervous system is preponderant in this phenomenon and its translation in terms of control of food intake by the central nervous system (CNS). Nutrient sensing by the extrinsic gastrointestinal nervous system may account for the satiation induced by food lipids, the satiety initiated by food protein, and for the rapid benefits of gastric bypass surgeries on both glucose and energy homeostasis. Thus, this recent knowledge provides novel examples of the mechanisms that control food intake and body weight, which might pave the way to future approaches in the prevention and/or treatment of obesity.

\section{Keywords:}

Extrinsic gastrointestinal nerves, satiation, satiety, macronutrients 
ROLE OF THE EXTRINSIC GASTROINTESTINAL NEURAL SYSTEM IN THE CONTROL OF HUNGER

The worldwide increase in obesity and its associated disorders makes the efforts to better understand the mechanisms that control food intake and energy homeostasis ever more crucial. The sensations of hunger and fullness are key determinants in the control of appetite and food intake. In normal individuals a precise balance exists between the sensation of hunger that precedes a meal and the sensation of fullness occurring after nutrient assimilation $^{1,2}$. Human and animal studies have suggested that this balance is deregulated in the context of obesity, whereby the sensation of fullness is inappropriately delayed or blunted, including that following lipid ingestion that has previously been associated with suppression of appetite and energy intake ${ }^{1,2}$. The mechanisms underlying the shift from the sensation of hunger to the sensation of fullness after a meal encompass the modulation of gastric distension, changes in gut motility and secretion of hunger stimulating or hunger suppressing gastrointestinal hormones. These hormones include the hunger stimulating peptide ghrelin, and peptides that reduce appetite such as cholecystokinin (CCK), peptide $\mathrm{YY}_{3-36}\left(\mathrm{PYY}_{3-36}\right)$ and glucagon-like peptide 1 (GLP1) (see ${ }^{3,4}$ for recent reviews). However, our understanding of the putative role of the gastrointestinal nervous system in the central or systemic effects of these hormones is limited. It is noteworthy that the effect of these gut derived hormones is blunted after surgical ablation of the vagal-brain stem neural communication ${ }^{5-8}$. Indeed, increases in food intake and growth hormone $(\mathrm{GH})$ secretion, both of which are promoted by ghrelin, are blunted after vagotomy ${ }^{5}$, as are the satiety effects initiated by $\mathrm{CCK}^{6}, \mathrm{GLP}^{7}$ or PYY $_{3-36}{ }^{7}$. Furthermore, the well established antihyperglycemic action of GLP1, a secretagogue that induces glucose stimulated insulin secretion (GSIS) and suppress glucagon secretion, is mediated at least in part via GLP1 receptors located in the periportal neural system. Accordingly, the improvement of glucose control by GLP1 is attenuated upon 
inhibition of the GLP-1 receptor in the portal vein ${ }^{8}$. Along these lines, it is of interest that recent data have highlighted the effect of macronutrients derived from food digestion in these processes. A rise in lipids, protein and glucose derived from carbohydrates, shortly after ingestion of a meal, activates the extrinsic gastrointestinal nervous system ${ }^{9-12}$, that alters appetite and the rate of hepatic glucose production in order to reduce appetite and maintain peripheral metabolic homeostasis. Such mechanisms may account for the rapid effects on hunger sensations and glucose control observed after gastric bypass surgery in rodents ${ }^{13,14}$. In this review, the mechanisms by which macronutrients are sensed by the extrinsic gastrointestinal neural system in relation to their early (satiation) or delayed (satiety) (see box 1) effect on food intake are discussed. Discussing these mechanisms is timely, especially because the knowledge of the nutrient effects on food intake has been updated recently.

\section{JEJUNAL SENSING AND EFFECTS OF LIPIDS}

It has long been accepted that lipids released from the digestion of fat-enriched meals exert satiation effects ${ }^{1,2}$. It was initially thought that the caloric load of lipids with regards to other macronutrients was a likely explanation for this phenomenon. Implementing this previous rationale, it was recently shown that the primary site of lipid sensing is restricted to the upper intestine ${ }^{17}$. This seminal study showed that increased long-chain fatty acyl-CoAs within the gut mucosa activate a gut-brain-liver neural axis that suppresses glucose production, an effect that is abolished after hepatic vagotomy, thus revealing a previously unrecognized regulatory pathway ${ }^{17}$. The mediator of this effect was shown to be the biologically active form of CCK, CCK-8, in the duodenum, and an active gut CCK-A receptor. It was subsequently deciphered that the downstream effector of the intestinal lipid metabolism initiating CCK secretion and action to lower hepatic glucose production through a neuronal network is duodenal protein kinase $\mathrm{C}$ delta $(\mathrm{PKC} \delta)^{18-20}$. Although these latter 
studies focused on the effects of jejunal lipid sensing (and/or CCK/ PKC $\delta$ ) on glucose production, and not on food intake, it is noteworthy to mention that unraveling the critical role of CCK in the lipid mediated effects on glucose homeostasis has provided a mechanistic rationale to the "satiation" action of lipids. It must be noted that a role for the membrane protein CD36 has also been suggested in the satiation effect of lipids. CD36 may act either as a transporter or a receptor of food lipids in the intestinal mucosa, thus serving as a molecular sensor that links fat ingestion to satiety ${ }^{21,22}$.

\section{PORTAL GLUCOSE ACTION ON FOOD INTAKE: SATIATION OR SATIETY?}

The rate of glucose appearance in the portal vein during the digestion of a carbohydrate-rich meal representative of current human nutrition (about $50 \%$ of calories as carbohydrates) is high. It may represent about one to two fold the equivalent of total endogenous glucose production (EGP) ${ }^{11}$ (Glossary). It has thus long been hypothesized that glucose might induce satiation in the course of meal digestion. To document this hypothesis, initial studies investigating the effect of glucose infusion into the portal vein on food intake and meal size in previously fasting rats were performed at rates matching EGP ${ }^{23,24}$. Glucose infusion into the portal vein at these rates induced a wide array of physiological and behavioral responses; a decrease in spontaneous food intake; an acquisition of food preference; and an alteration of the electrical activity of hepato-portal vagal and spinal afferents, and of hypothalamic neurons implicated in the control of appetite (see ${ }^{25}$ for a review). However, portal glucose infusion at much lower rates (one sixth to one third of EGP) is sufficient to initiate both a limitation of food intake and the activation of hypothalamic nuclei, as revealed by C-FOS labeling, in rats ${ }^{11,26}$. Whilst either low or high rates of portal glucose infusion might represent what occurs during the postprandial period, various arguments have suggested that the portal delivery of glucose does not determine the 
termination of an ongoing meal but, instead, drives the size of the following meal ${ }^{27}$. This has suggested that portal glucose sensing might be related to satiety, rather than to satiation. In agreement with the latter rationale, it is noteworthy that the portal region has also been demonstrated to be critical for the detection of slowly induced hypoglycemia ${ }^{28}$, similar to the transition from a post-absorptive to a fasted state. Along these lines a question that arises is whether or not portal glucose appears under post-absorptive conditions.

\section{MOLECULAR MECHANISM OF PORTAL GLUCOSE SENSING}

Recently, molecular pathways involved in the sensing of glucose appearance at low rates in the portal vein were investigated, in rodents ${ }^{11}$. Three potential candidate mechanisms, which could account for different types of glucose sensing, were considered: (i) the low affinity glucose transporter Glut 2 which is coupled to glucokinase-induced glucose phosphorylation and may account for GSIS by pancreatic $\beta$ cells in mice ${ }^{29}$; (ii) gut taste receptors. similar to those in the tongue, that respond to sweet, bitter, umami, and fatty acids and together with other chemosensory signals may coordinate the release of hormones that regulate glucose homeostasis $^{30}$; and (iii) the sodium-coupled glucose co-transporter 3 (SGLT3), a member of the SGLT family of transport proteins that includes SGLT 1 and 2, a receptor (not a transporter) of glucose, which is responsible for glucose-stimulated secretions by enterochromaffin cells ${ }^{31}$. A body of evidence points to a critical role for SGLT3, rather than for Glut2-glucokinase or taste receptors, in the sensing of glucose appearance at low physiological rates in the portal vein walls which include the inhibition of portal glucose sensing by phloridzin (a specific inhibitor of SGLTs), the activation of portal glucose sensing by $\alpha$-methyl-glucose (a non-phosphorylable analog of glucose), which is transported by SGLT 1 and 2 and binds to SGLT3, and the absence of activation by 3-0-methylglucose, which is transported by SGLT1 and 2, but does not bind to SGLT3 ${ }^{11}$. It must be noted that 
afferents innervating the portal vein may travel via both the common hepatic branch and the celiac branch of the vagus nerve, but also via the dorsal root spinal way ${ }^{32}$. It is noteworthy that the portal glucose signal was not ablated by surgical vagotomy of the common hepatic branch ${ }^{11}$. This suggests that the signal might also be conveyed either by the vagal celiac branch or the spinal way, whereas a widely acceptable view is that it should be conveyed by the ventral vagus ${ }^{11}$. Therefore, SGLT3, and not Glut2 or taste receptors, is likely to be the sensor initiating the portal glucose signal conveyed to the brain to suppress appetite.

\section{ROLE OF PORTAL GLUCOSE SENSING IN THE SATIETY EFFECT OF FOOD}

\section{PROTEIN}

The mechanism by which dietary proteins exert their satiety effect is not clear. An interesting hypothesis was derived from the observation that diets deficient in one essential amino acid, e.g. leucine, rapidly reduce food intake in animals due to an innate aversion to diets with imbalanced amino acid composition (see ${ }^{33,34}$ for recent reviews). The mechanism underlying sensing of imbalanced amino acid diets was shown to involve phosphorylation of the translation initiation factor $2 \alpha$ (eIF2alpha) by the ubiquitous kinase GCN2, in the anterior pyriform cortex of the brain, in response to intracellular amino acid deficiency. Importantly, inactivation of the kinase impaired this aversive response ${ }^{35,36}$. Therefore, this intracellular signal transduction pathway activates a neuronal circuit that promotes rejection of imbalanced food sources. Vagotomy experiments have also suggested a role for the autonomic efferent nervous system in the reinforcement of the effect ${ }^{37}$. However, a putative role for the extrinsic gastrointestinal afferents in the primary sensing of imbalanced diets has not been established yet (see "outstanding question" box).

It has been hypothesized that diets enriched in a given source of protein could initiate a weak decrease in food intake because of the potential imbalance in their content in amino 
acids. However, this hypothesis is not supported by experimental evidence. Two related hypotheses with regards to the hunger-curbing effects of protein-enriched diets suggested that the decreased food intake could be due to a conditioned taste aversion, driven by either malaise or low palatability of the diet. However, both hypotheses have been ruled out ${ }^{26,38}$. A putative role of amino acid taste receptors in sensing imbalanced diets ${ }^{30}$ has also been ruled out. Indeed, mice null for the transient receptor potential melastin 5 (Trmp5), a protein required for the transmission of the taste receptors signal, retained their sensitivity to the satiety action of protein-enriched diets ${ }^{11}$. The role of the hypothalamic melanocortin system, a collection of central nervous system circuits, which include orexigenic and anorexigenic neuropeptides, has also been investigated. In this context, protein enriched diets had an opposing effect and promoted an increase, rather than a decrease, in the orexigenic peptide agouti-related protein (AGRP) and a decrease in the anorexigenic alpha melanocortinstimulating hormone ( $\alpha-\mathrm{MSH})$. This suggests that the melanocortin system does not mediate the hunger-curbing effects of protein enriched diet and instead, it might function to defend the body against variations in food intake generated by the nutritional environment ${ }^{39}$.

However, protein-enriched diets might initiate their satiety effects indirectly, via glucose sensing. During their digestion, proteins induce the expression of gluconeogenic genes in the gut, such as the genes encoding for glucose-6 phosphatase catalytic subunit (G6PC), phosphoenolpyruvate carboxykinase-cytosolic form (PEPCK-C) and glutaminase (see ${ }^{25,40}$ for recent reviews), thus resulting in the release of glucose into the portal vein, in the post-absorptive period. Compared to the $5-10 \%$ of total EGP seen after a standard carbohydrate-enriched meal, intestinal gluconeogenesis accounts for as much as $20-25 \%$ of EGP after a protein-enriched diet ${ }^{26}$. This is enough to counterbalance the intestinal glucose uptake, so that portal glycemia is equal to arterial glycemia in the post-absorptive state (portal glycemia is lower than arterial glycemia in the post-absorptive state after carbohydrate diets) 
26. This is sufficient to activate the portal glucose sensor and to curb hunger $25,26,40$. As expected, portal innervation is essential in this phenomenon since a local peri-portal treatment with capsaicin (a drug inactivating both vagal and spinal afferents) abolishes the satiety effect induced by protein diets ${ }^{26}$. Mice with specific deletion of G6PC in the intestine are insensitive to the satiety induced by protein-enriched diets, thus confirming a causal link in the satiety effect of dietary protein ${ }^{41}$.

Thus, proteins act indirectly by the means of intestinal gluconeogenesis and portal glucose sensing to curb food intake.

\section{SENSING OF PROTEIN DIGESTS AND INDUCTION OF GUT GLUCONEOGENESIS}

Mu-opioid receptors (MORs) expressed in the brain can interfere with the control of food intake, via their role in the "reward" system ${ }^{42,43}$. It has long been known that proteolytic moieties released from alimentary protein exhibit $\mu$-opioid activity in vitro ${ }^{44}$. In line with this, it is well established that oligopeptides of variable size that exhibit $\mu$-opioid activity require a minimal structure of a dipeptide ${ }^{45-47}$. Oligopeptides deriving from the digestion of alimentary protein may be delivered in the portal blood ${ }^{48}$. However, it is unlikely that they reach the brain, because they are metabolized in the liver. It has thus been proposed that these oligopeptides might exert a signaling activity at a gastrointestinal or mesenteric site, activating MORs present in the enteric neural system ${ }^{49}$. This has raised the attractive hypothesis that sensing of oligopeptides deriving from protein digestion by MOR might be involved in the control of food intake via intestinal gluconeogenesis ${ }^{12}$. In line with this, MOR-agonists, such as the synthetic, high affinity opioid peptide DAMGO, suppress intestinal gluconeogenesis genes expression and increase food intake when they are infused in the portal vein of conscious rats. On the contrary, MOR-antagonists (such as naloxone) or various protein derived oligopeptides induce intestinal gluconeogenesis and blunt food intake 
12. The brain regions receiving inputs of both the ventral vagal afferents (i.e. the dorsal vagal complex) or the spinal afferents (i.e. the parabrachial nucleus) are involved in the reflex arc regulating gut gluconeogenesis. All these effects are dependent on the integrity of the periportal nervous system ${ }^{12}$. That MOR-dependent induction of intestinal gluconeogenesis is causal in the decreased hunger promoted by protein-diets was indicated by the observation that: 1) MOR-knockout mice do not induce intestinal gluconeogenesis in response to oligopeptides and are insensitive to protein-enriched diets; 2) mice with intestinal G6pc gene deletion do not decrease food intake in response to portal infusions of MOR-antagonists or oligopeptides $^{12}$.

It must be underlined that the induction of intestinal gluconeogenesis by dietary protein is dependent on gene expression, that takes place over the postprandial period. After that, portal glucose appearance may initiate portal glucose sensing and drive central satiety (fig 1). This can last after the postprandial time, since it is dependent on enzyme induction ${ }^{12}$, ${ }^{26}$. Hence, deciphering the role of intestinal gluconeogenesis in the decrease of food intake induced by protein-enriched diets has provided both a mechanistic explanation for the satiety (as opposed to satiation) effect of food protein and a physiological meaning to the hungercurbing effect of portal glucose-sensing.

\section{WHICH MECHANISMS OPERATE IN OBESITY SURGERY?}

Gastric bypass surgery has shown impressive outcomes in the control of overall glucose homeostasis, even before the substantial weight loss following surgery of morbidly obese diabetic patients ${ }^{50-52}$. It has been suggested that the decreased hunger sensation and the rapid amelioration of diabetes after gastric surgery might be mediated by an increased secretion of GLP-1 and consecutively insulin ${ }^{50-52}$. However, an alternative explanation, at least in a model of gastric bypass in mice, is that induction of intestinal gluconeogenesis 
relayed by portal glucose sensing is causal to these phenomena ${ }^{13}$. Along these lines, a comparable increase in insulin sensitivity of EGP, with induction of gluconeogenic genes in the kidney, was observed when intestinal gluconeogenesis was induced by protein-rich diets, in rats ${ }^{53}$. On the other hand, a study by Hayes et al provided no direct evidence to support the hypothesis that intestinal gluconeogenesis contributes to the resolution of T2D seen after gastric bypass of obese and diabetic patients, when compared to obese non diabetic patients 54. The authors interpreted the net increase in portal glucose concentration observed in the non-diabetic subjects as weak ${ }^{54}$. However, the role of intestinal blood flow, which is known to be very high ${ }^{55}$, was not taken into consideration, in this study. Taking into account intestinal blood flow and the increase in portal glucose, a more careful interpretation might suggest that intestinal glucose production after gastric bypass in the non-diabetics is no less than $25 \%$ of the total glucose production of the body ${ }^{55}$. Moreover, in the diabetic subjects, the portal glucose concentration was equal to that of the peripheral glucose concentration ${ }^{55}$. This has been shown to be sufficient, at least in rodents, in activating portal glucose sensing and in promoting its benefits on glucose and energy homeostasis ${ }^{13,26,53}$. Finally, it is noteworthy that the beneficial effects of intestinal gluconeogenesis on insulin sensitivity, and of GLP 1 on insulin secretion, may act in synergy to account for the whole systemic effect of bypass on glucose control ${ }^{56}$. A pending question in this area is why and how intestinal gluconeogenic genes expression is induced after gastric bypass. Since MORs are widely expressed along the gut $^{49}$, the possibility that they might be involved in this induction in relation with the protein content of the diet is attractive and warrants careful testing (see "Outstanding Questions").

As outlined above, the benefits of portal glucose sensing may operate during the postabsorptive period (time of occurrence of satiety) rather than during the postprandial period (time of occurrence of satiation). Whether gastric bypass might also induce a process compatible with the time of "satiation", i.e. deriving directly from the digestion of nutrients, 
deserves further investigation. Indeed, it was recently shown that a jejunal lipid-sensing mechanism might account for the effect of gastric bypass on glucose control in rats ${ }^{14}$. Interestingly, in the situation of gastric bypass, glucose absorption might concur to the effects observed on glucose control ${ }^{14}$. Again, the mechanistic chain of events involves the extrinsic gastrointestinal nervous afferents ${ }^{14}$.

It is intuitive that the combination of mechanisms taking place during the postprandial time (lipid sensing) ${ }^{14}$, the satiety time (intestinal gluconeogenesis) ${ }^{13}$, and of GLP1-driven effects ${ }^{56}$, might fully account for the dramatic amelioration of obesity and glucose control after gastric bypass surgery. However, how jejunal lipid sensing and intestinal gluconeogenesis cooperate to exert their effects is an open question.

\section{CONCLUDING REMARKS AND FUTURE PERSPECTIVES}

That the brain, especially the hypothalamus, plays a major role in integrating various peripheral signals (e.g. hormones such as leptin or insulin) to influence food intake and energy expenditure has been widely accepted for some time ${ }^{57}$. Now, peripheral sites such as gastrointestinal neural system are also implicated as being crucial for the control of hunger sensation or glucose homeostasis. Three major macronutrients derived from food digestion (lipid, glucose, protein) may control hunger sensations via their intestinal sensing from the gut, and working in harmony with the brain might integrate these signals to influence hunger and endogenous glucose control ${ }^{1,2,13,14,53}$. The vagal way is often thought to play a major role in conveying the signals from the extrinsic neural system to the brain ${ }^{25,32}$. However, the participation of the spinal way has been evidenced for the portal glucose signalling ${ }^{11}$ or the protein signalling ${ }^{12}$. It remains to be seen whether specific signals may travel along the one or the other way, respectively. Another interesting point is that portal glucose has been associated with the initiation of a food preference ${ }^{23,25}$. The acquisition of food preference is 
notably centrally biased by the reward system ${ }^{42,43}$. To date, the impact of the portal glucose signal has been characterized in the hypothalamus ${ }^{25,26}$. The hypothesis of an anatomical and/or functional impact of portal glucose in brain regions implicated in the reward system, e.g. the accumbens nucleus, deserves further investigation (see "Outstanding Questions").

Recent progress has allowed us to understand better how macronutrients signal to the brain to decrease appetite and food intake, and how important are the extrinsic nerves of the gastrointestinal system in sensing and conveying these signals to the brain. Much remains to be known relating to the specificities underlying the mechanisms initiated by lipids, proteins or glucose, especially regarding to their routing and their brain targets. Continuing efforts is warranted, since the future knowledge could offer novel paradigms of food intake control, which may provide as much as novel approaches of treatment and/prevention of metabolic diseases.

\section{OUSTANDING QUESTIONS}

- Does the extrinsic gastrointestinal nervous system have a role in the primary sensing of amino-acid imbalanced diets?

- What are the mechanisms underlying the induction of gluconeogenic gene expression after gastric bypass?

- Is there a specific role for $\mu$-opioid receptors in the induction of intestinal gluconeogenesis after bypass?

- How do jejunal lipid sensing and intestinal gluconeogenesis cooperate towards improving glucose fluxes and and reducing food intake after gastric bypass?

- Do specific signals travel along the vagal or the spinal routes of the extrinsic gastrointestinal neural system, respectively? 
- Does the portal glucose signal modulate the activity of the brain regions involved in the reward system?

\section{TEXT BOX 1: Satiation or satiety: a question of definition and time of occurrence}

There is often confusion in the use of the words "satiation" and "satiety". "Satiation" is defined as the sensation of fullness, which progressively takes place during the absorption and digestion of the meal. In other words, satiation derives from the sum of the mechanisms, which take place during the digestion of nutrients and concur to curb the sensation of hunger. $\mathrm{CCK}$, which is secreted early in the blood in response to the meal and decreases hunger, is a typical hormone of satiation ${ }^{15}$. In contrast, "satiety" is defined as a state of no-hunger, taking place sometime after the last meal. Thus, satiety operates to moderate the hunger sensation at a time of initiation of the following meal. For instance, it has long been known that alimentary protein induces a satiety, and not satiation, phenomenon ${ }^{16}$. It must be mentioned that it is sometimes difficult to ascribe a role of "satiation" or "satiety" to a given mechanism. For instance, CCK may have a delaying effect on the return of hunger sensations via its action of inhibition of gastric emptying ${ }^{1}$. The notions of satiation and satiety are thus defined in a context of physiology. Time constraints are often mandatory in certain experimental contexts, e.g. hyperinsulinemic euglycemic clamp studies or brain C-FOS labeling determinations. The data obtained from such experiments must therefore be reintegrated in the physiological context with precautions, as far as their interpretation in terms of "satiation" or "satiety" phenomenon is concerned.

\section{GLOSSARY}


C-FOS labeling: a method based on the immunohistochemical identification of the protein C-FOS, an established marker of neuronal activation. The method is not specific and requires

328 that the analysis is performed in absence of any other signals capable of affecting the activity of neurons in the same area.

Endogenous glucose production (EGP): a crucial physiological function, which allows the body to maintain plasma glucose concentration around $1 \mathrm{~g} / \mathrm{L}$ in the absence of glucose supply from food (post-absorptive and fasting periods). EGP may proceed from a pre-formed store of glucose (glycogen) in the liver or from gluconeogenesis (see below) in the liver, kidney and intestine. These three organs only can contribute to EGP, since they are the only organs known to express glucose- 6 phosphatase, the enzyme in the last step in the biochemical reaction preceding glucose release ${ }^{25}$.

Extrinsic gastrointestinal nervous system: refers to the nerves spreading in the abdomen, which include the common hepatic and the celiac branches of the vagal nerve connected to the CNS and the sympathetic nerves connected to the spinal cord ${ }^{32}$.

Gluconeogenesis: refers to the synthesis of glucose from non-glucidic carbon compounds. Gluconeogenesis becomes essential for EGP when liver glycogen stores are exhausted, such as during fasting. The main glucose precursors are lactate and alanine in the case of liver gluconeogenesis, whereas glutamine is an essential substrate for gluconeogenesis in the kidney and intestine ${ }^{25}$.

Glucose stimulated insulin secretion (GSIS): refers to the fraction of insulin, which is released in response to an increase in blood glucose concentration and adds to the basal insulin release. Intrinsic gastrointestinal nervous system: refers to the nerves closely surrounding the gut mucosa (the so-called Meisner's plexus) and those of the gut muscular layer (the so-called Auerbach's plexus). 
Hyperinsulinemic euglycemic clamp: a method based on the use of metabolic tracers of

351

352

353

354

355

356

357

358

359

360

361

362

363 glucose to quantify endogenous glucose fluxes (production and utilization) and/or the action of insulin to suppress glucose production and/or enhance glucose utilization. It must be carried out in the absence of glucose appearing from food origin-

Melanocortin system: a major system expressed in the hypothalamus that regulates hunger sensations. It notably comprises the melanocortin receptor type 4 (MC4R) and two main neuro-mediators able to control its activity: alpha-melanin stimulating hormone $(\alpha-\mathrm{MSH})$ is an MC4R agonist and allows MC4R to inhibit hunger, agouti-related protein (AgRP) is an antagonist and activates hunger. Insulin or leptin mediate their anorexigenic effect via the release of a-MSH, whereas ghrelin mediates its orexigenic effect via the activation of AgRP release. See ${ }^{39}$ as a recent review.

M-opioid receptor (MOR): receptors of opioids from exogenous (e.g. morphin) or endogenous (endorphins) origin. In the brain, MOR may participate to the control of food intake, especially via their implication in the so-called "reward" system ${ }^{28,29}$. Agonists like morphine stimulate hunger, whereas antagonists like naloxone decrease hunger. In the gut, they were shown to control motility processes ${ }^{31}$.

\section{REFERENCES}

1. Covasa M. (2010) Deficits in gastrointestinal responses controlling food intake and body weight. Am J Physiol Regul Integr Comp Physiol 299, R1423-R1439.

2. Little T.J. and Feinle-Bisset C. (2011) Effects of dietary fat on appetite and energy intake in health and obesity--oral and gastrointestinal sensory contributions. Physiol Behav 104, 613620.

3. Yi C.X. et al. (2010) The role of the autonomic nervous liver innervation in the control of energy metabolism. Biochim Biophys Acta 1802, 416-431.

4. Janssen P. et al., (2011) Review article: the role of gastric motility in the control of food intake. Aliment Pharmacol Ther 33, 880-894.

5. Date Y. et al. (2002) The role of the gastric afferent vagal nerve in ghrelin-induced feeding and growth hormone secretion in rats. Gastroenterology 123, 1120-1128.

6. Smith G.P. et al. (1981) Abdominal vagotomy blocks the satiety effect of cholecystokinin in the rat. Science 213, 1036-1037. 
7. Abbott C.R. et al. (2005) The inhibitory effects of peripheral administration of peptide YY(336) and glucagon-like peptide-1 on food intake are attenuated by ablation of the vagalbrainstem-hypothalamic pathway. Brain Res 1044, 127-131.

8. Vahl T.P et al. (2007) Glucagon-like peptide-1 (GLP-1) receptors expressed on nerve terminals in the portal vein mediate the effects of endogenous GLP-1 on glucose tolerance in rats. Endocrinology 148, 4965-4973.

9. Breen D.M. et al. (2011) Gut-brain signalling: how lipids can trigger the gut. Diabetes Metab Res Rev 27, 113-119.

10. Rasmussen B.A. et al. (2012) Lipid sensing in the gut, brain and liver. Trends Endocrinol Metab 23, 49-55

11. Delaere F. et al. (2012) The role of sodium-coupled glucose co-transporter 3 in the satiety effect of portal glucose sensing. Mol Metab doi: 10.1016/j.molmet.2012.11.003 http://www.elsevier.com/journals/molecular-metabolism/2212-8778

12. Duraffourd C. et al. (2012) Mu-opioid receptors and dietary protein stimulate a gut-brain neural circuitry limiting food intake. Cell 150,377-388

13. Troy S. et al. (2008) Intestinal gluconeogenesis is a key factor for early metabolic changes after gastric bypass but not after gastric lap-band in mice. Cell Metab 8, 201-211.

14. Breen D.M. et al. (2012) Jejunal nutrient sensing is required for duodenal-jejunal bypass surgery to rapidly lower glucose concentrations in uncontrolled diabetes. Nat Med 18, 950955.

15. Gibbs J. et al. (1976) Cholecystokinin-decreased food intake in rhesus monkeys. Am J Physiol 230, 15-18.

16. Rolls B.J. et al. (1988) The specificity of satiety: the influence of foods of different macronutrient content on the development of satiety. Physiol Behav 43, 145-153.

17. Wang P.Y. et al. (2008) Upper intestinal lipids trigger a gut-brain-liver axis to regulate glucose production. Nature 452, 1012-1016.

18. Cheung G.W. et al. (2009) Intestinal cholecystokinin controls glucose production through a neuronal network. Cell Metab 10, 99-109.

19. Kokorovic A. et al. (2011) Duodenal mucosal protein kinase $C-\delta$ regulates glucose production in rats. Gastroenterology 141, 1720-1727.

20. Breen D.M. et al. (2011) Duodenal PKC- $\delta$ and cholecystokinin signaling axis regulates glucose production. Diabetes 60, 3148-3153.

21. Naville D. et al. (2012) Linik beetween intestinal CD36 ligand binding and satiety induced by a high protein diet in mice. PlosONE 7, e30686.

22. Schwartz G.J. et al. (2008) The lipid messenger OEA links dietary fat intake to satiety. Cell Metab 8, 281-288.

23. Tordoff M.G. and Friedman M.I. (1986) Hepatic portal glucose infusions decrease food intake and increase food preference. Am J Physiol 251(1 Pt 2), R192-R196.

24. Langhans W. et al. (2001) Intrameal hepatic-portal infusion of glucose reduces spontaneous meal size in rats. Physiol Behav 73, 499-507.

25. Delaere F. et al. (2010) Hypothalamic integration of portal glucose signals and control of food intake and insulin sensitivity. Diabetes Metab 36, 257-262.

26. Mithieux G. et al. (2005) Portal sensing of intestinal gluconeogenesis is a mechanistic link in the diminution of food intake induced by diet protein. Cell Metab 2, 321-329.

27. Baird J.P. et al. (1997) Intake suppression after hepatic portal glucose infusion: all-or-none effect and its temporal threshold. Am J Physiol 272(5 Pt 2), R1454-R1460.

28. Saberi M. et al. (2008) The locus for hypoglycemic detection shifts with the rate of fall in glycemia: the role of portal-superior mesenteric vein glucose sensing. Diabetes 57, 13801386.

29. Thorens B. and Larsen, P.J. (2004) Gut-derived signaling molecules and vagal afferents in the control of glucose and energy homeostasis. Curr Opin Clin Nutr Metab Care 7, 471-478.

30. Janssen S. and Depoortere, I. (2012) Nutrient sensing in the gut: new roads to therapeutics? Trends Endocrinol Metab doi: 10.1016/j.tem.2012.11.006

http://www.cell.com/trends/endocrinology-metabolism 
31. Freeman S.L. et al. (2006) Luminal glucose sensing in the rat intestine has characteristics of a sodium-glucose cotransporter. Am J Physiol Gastrointestinal and Liver Physiology 8, 532 539.

32. Berthoud H.E. (2004) Anatomy and function of sensory hepatic nerves. Anat Rec Discov Mol Cell Evol Biol 280, 827-835.

33. Chaveroux C., et al. (2010) Molecular mechanisms involved in the adaptation to amino acid limitation in mammals. Biochimie 92, 736-745.

34. Anthony T.G. and Gietzen D.W. (2013) Detection of amino acid deprivation in the central nervous system. Curr Opin Clin Nutr Metab Care 16, 96-101.

35. Maurin A.C. et al. (2005) The GCN2 kinase biases feeding behavior to maintain amino acid homeostasis in omnivores. Cell Metab 1, 273-277.

36. Hao S. et al. (2005) Uncharged tRNA and sensing of amino acid deficiency in mammalian piriform cortex. Science 307, 1776-1778.

37. Bellinger L.L. et al. (2005) Autonomic efferents affect intake of imbalanced amino acid diets by rats. Pharmacol Biochem Behav 81, 24-31.

38. L'Heureux-Bouron D. et al. (2004) A very high 70\%-protein diet does not induce conditioned taste aversion in rats. $J$ Nutr $134,1512-1515$.

39. Pillot B. et al. (2011) Role of hypothalamic melanocortin system in adaptation of food intake to food protein increase in mice. PlosONE 6, 1-7.

40. Mithieux, G. et al. (2009) Intestinal gluconeogenesis : key signal of central control of energy and glucose homeostasis. Curr Opin Clin Nutr Metab Care 12, 419-423.

41. Penhoat, A. et al. (2011) Protein-induced satiety is abolished in the absence of intestinal gluconeogenesis. Physiol Behav 105, 89-93.

42. Berridge C.W. (2009) 'Liking' and 'wanting' food rewards: brain substrates and roles in eating disorders. Physiol Behav 97, 537-550.

43. Berridge C.W. et al. (2010) Hypocretin/orexin in arousal and stress. Brain Res 1314, 91-102.

44. Zioudrou C. et al. (1979) Opioid peptides derived from food proteins. The exorphins. J Biol Chem 254, 2446-1449.

45. Capasso A. et al. (1997) Design of mu selective opioid dipeptide antagonists. FEBS Lett 417, 141-144.

46. Moritoki H. et al. (1984) Tripeptides acting on opioid receptors in rat colon. Eur J Pharmacol 100, 29-39.

47. Schiller P. et al. (2002) Opioid dipeptide derivatives with a mixed $\mu$ antagonist $/ \delta$ antagonist, partial $\mu$ agonist $/ \delta$ antagonist or $\mu$ agonist/partial $\delta$ agonist profile. American Peptide Symposia 6, 229-270.

48. Lee V.H. (2000) Membrane transporters. Eur J Pharm Sci. Suppl 2, S41-S50.

49. Holzer P. (2009) Opioid receptors in the gastrointestinal tract. Regul Pept 155, 11-17.

50. Thaler J.P. and Cummings, D.E. (2009) Minireview: Hormonal and metabolic mechanisms of diabetes remission after gastrointestinal surgery. Endocrinology 150, 2518-2525.

51. Sala P.C. et al. (2012) Type 2 diabetes mellitus: a possible surgically reversible intestinal dysfunction. Obes Surg 22, 167-176.

52. Perez-Tilve D. et al. (2008) A sweet spot for the bariatric surgeon. Cell Metab 8, 177-179.

53. Pillot B. et al. (2009) Protein feeding promotes redistribution of endogenous glucose production to the kidney and potentiates its suppression by insulin. Endocrinology 150, 616624.

54. Hayes M.T. et al. (2011) Is intestinal gluconeogenesis a key factor in the early changes in glucose homeostasis following gastric bypass? Obes Surg 21, 759-762.

55. Mithieux G. (2011) Comment about intestinal gluconeogenesis after gastric bypass in human in relation with the paper by Hayes et al., Obes. Surg. 2011. Obes Surg 22, 1920-1922.

56. Mithieux G. (2012) A synergy between incretin effect and intestinal gluconeogenesis accounting for the rapid metabolic benefits of gastric bypass surgery. Curr Diab Rep 12, 167 171.

57. Warne, J.P. and Xu, A.W. (2012) Metabolic transceivers: in tune with the central melanocortin system. Trends Endocrinol Metab doi: 10.1016/j.tem.2012.10.005; http://www.cell.com /trends/endocrinology-metabolism 
58. Croset M. et al. (2001) Rat small intestine is an insulin-sensitive gluconeogenic organ. Diabetes 50, 740-746.

59. Mithieux G. et al. (2004) A novel role for glucose 6-phosphatase in the small intestine in the control of glucose homeostasis. J Biol Chem 279, 44231-44234.

\section{FIGURE LEGEND:}

Figure 1: Sequential events controlled by food protein and glucose via the gastrointestinal neural network

A) During the postprandial period, peptides released in the portal vein from protein digestion binds to and antagonize MORs present in the peri-portal afferents. The MOR-controlled ascending nerves convey the message to the brain nuclei connecting the afferents from either the vagal or the spinal way. A reflex arc drives the induction of the intestinal regulatory gluconeogenesis genes.

B) The intestinal gluconeogenic pathway, stimulated in response to the induction of glutaminase, PEPCK-C and G6Pase activities, efficiently converts glutamine (the main intestinal glucose precursor) and glycerol (an accessory glucose precursor) into glucose. See ref $^{58,59}$ for a comprehensive description of these pathways.

C) During the post-absorptive period, glucose is released in the portal vein and sensed by SGLT3, which sends a second message to the brain to decrease hunger at the hypothalamic site and increase insulin sensitivity of hepatic glucose production via a second reflex arc. 